\title{
Energy Management System for Offshore Wind Farms
}

\author{
Ch. Eping, J. Stenzel \\ Institute of Electrical Power Systems \\ TU-Darmstadt \\ Landgraf-Georg-Straße 4 \\ 64283 Darmstadt \\ Phone number: +49 6151 16-6586, +49 6151 16-2852 \\ e-mail: christiane.eping@eev.tu-darmstadt.de, juergen.stenzel@eev.tu-darmstadt.de
}

\begin{abstract}
This paper presents an energy management system (EMS) especially for offshore wind farms. The main idea of the EMS is to reduce the impact of wind energy on the system. Therefore a power schedule to reduce the fluctuating impact on the power system is developed. This power schedule afford as well an optimisation for the maintenance of the windmills. The maintenance interval optimisation is therefore also included in the EMS. The EMS has also the function to shutdown the wind farms in case of to much wind. It is obvious that a well-organised procedure for reducing the infeed power into the high-voltage system is required. The transmission system operator (TSO) is informed in advance about the power schedule and about the shutdown schedule. The main objective of the TSO is to minimize the impact on the power quality. For that he has to organize sufficient regulating power.
\end{abstract}

\section{Key words}

energy management, offshore wind farm, power control, shutdown, power schedule

\section{Introduction}

Wind energy is one of the most increasing electricity sources of the 21st century. It is expected that a large amount of wind energy converters, especially offshore will be installed in the near future.

With the increase of wind power production, i.e. the rising offshore wind farms, questions regarding the impact on the conventional generation system are gaining importance[1]. In the near future offshore wind farms with a capacity of about $500 \mathrm{MW}$ to $1000 \mathrm{MW}$ will be created. These are dimensions of actual base load power plants. In this paper an energy management system is presented. The energy management system (EMS) is a control system for a whole wind farm with the aim to reduce the fluctuating energy fed-in by the offshore wind farms. Therefore two main tasks are included in the EMS:

- power schedule

- shutdown schedule
A power schedule for offshore wind farms is presented. Such a power schedule can be received by the TSO e.g., from industrial consumers in order to make a good unit commitment. Here it is expanded on fluctuating power producers, especially wind energy.

A shutdown schedule for an offshore wind farm is developed. According to the increase of the capacity of large wind farms, it is difficult for the TSO to react properly in case of a storm caused shutdown. Usually a windmill will be shutdown automatically when its maximum wind speed is exceeded. A sudden and uncontrolled shutdown of a wind farm with an installed capacity of about $1000 \mathrm{MW}$ is comparable with an unexpected disconnection of a base load power plant.

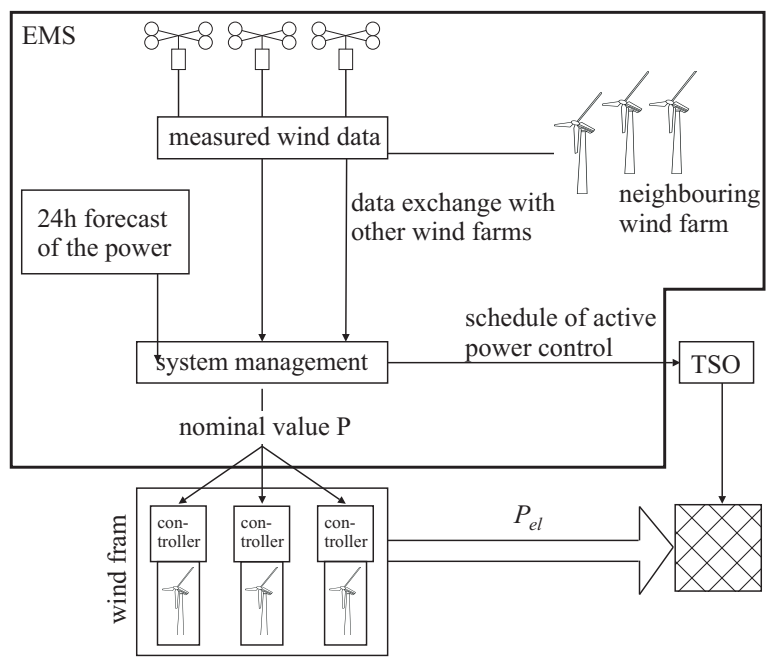

Fig. 1 Energy Management System (EMS)

In Figure 1 the data flow of the EMS is shown. The daily power forecast as well as the measured wind data are the basic input data for the system management. Both schedules for the power are developed by the system management on the base of the input data. The information about the schedule is given to the TSO.

In addition to the EMS every single windmill has a currently used control system (UCS) $[2,3]$ which is 
primary power controlled. In the main the EMS arranges the nominal value $\mathrm{P}$ for the UCS. This nominal value for the power $\mathrm{P}$ depends on the calculated schedule. Except, in case of exceptional circumstances, the instructions given by the EMS can be ignored. Exceptional circumstances might be for example emergency shutdowns.

\section{Power schedule}

The power schedule emanate from the daily power forecast [6]. According to the power schedule demanded of the German industries the interval size is at least a quarter hour. Also this schedule has to be calculated once a day and given to the TSO. The schedule is always for the next day. Especially wind energy is fluctuating very much so it makes sense to develop a schedule for the TSO. The advantage of a power schedule is obvious: The unit commitment for the next day will be improved.

\section{A. Power schedule calculation}

The base for the power schedule is the daily power forecast. This forecast has a certain frequency distribution. According to the daily power forecast and the frequency distribution a schedule for the wind farm is developed.

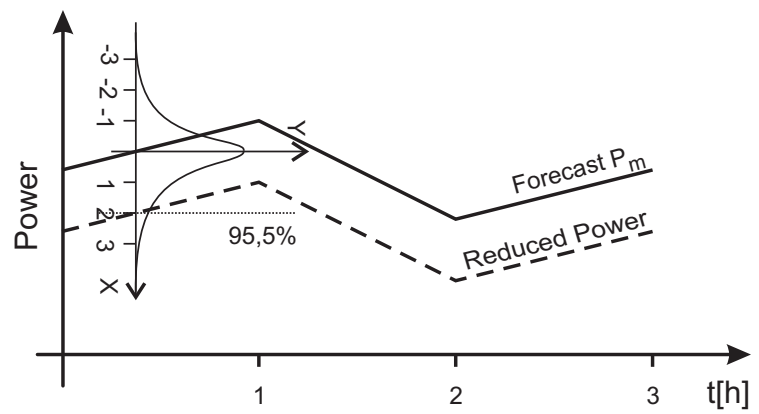

Fig. 2 Getting the reduced power curve

First of all, depending on the demanded forecast accuracy, the power curve is shifted as shown in Figure 2. Therefore the frequency distribution is drawn on.

$$
y=\frac{1}{\sigma \sqrt{s \pi}}^{-\frac{x^{2}}{2 \sigma^{2}}}
$$

The frequency distribution and the required reliability of the power schedule is decisive for the margin between the forecasted power and the scheduled power. Here, as an example, a probability of

$$
p(|x|<2)=95,5 \%
$$

is assumed [4].

Intervals of constant power are created under the reduced power curve (Figure 3 ). The size of the intervals for the power schedule is limited by the forecast which is given in one hour intervals. There is no exact upper limitation for the interval size, but the interval size should be determined with the objective of using as much energy as possible. Of course the interval size depends on the characteristic of the daily power forecast. Following the power schedule for the German industries, the size of the power steps can be varied in quarters of an hour.

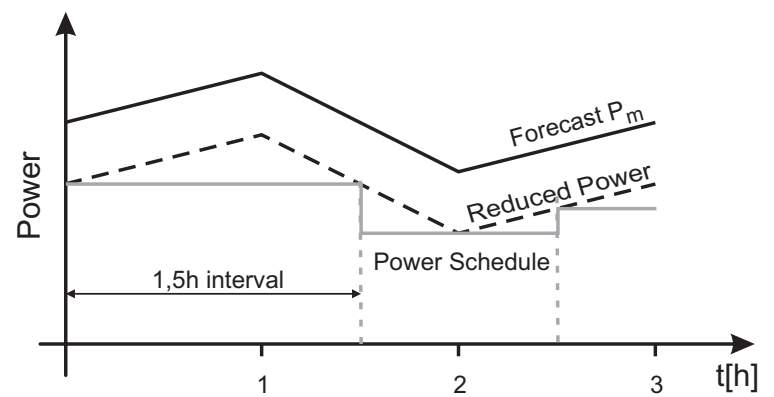

Fig. 3 The power schedule

The optimisation for the power schedule is solved by a genetic algorithm. This algorithm can optimise the exploitation of the energy while maintaining also the other conditions. The genetic algorithm is the natural tool for solving the optimization problem when it does not lead itself to an easy analytical treatment [4].

The result is a nearly optimal power schedule for the next day.

\section{B. Maintenance Interval Optimisation (MIO)}

The maintenance interval optimisation (MIO) organises the in service hours of all the windmills in a wind farm. The aim of this organisation is to have the maintenance of all windmills in one time period to avoid extra journeys on the sea.

In detail the MIO calculates how many windmills have to operate during a period of the power schedule. The MIO is an additional procedure and needs the power schedule as well as the forecast as input data.

As it can bee seen as hatched areas in Figure 4 the rated power $P_{r w}$ of the wind farm can not be generated because of less wind. This gives the information about the maximum power $P_{m}$ a windmill can generate at each moment. The grey area in Figure 4 is the power that will not be exploited. Hence not all turbines have to be in service.

The decision which turbines will run, pleases the following criteria:

- Required power.

- Attention of shadowing effects.

- Number of in service hours. 


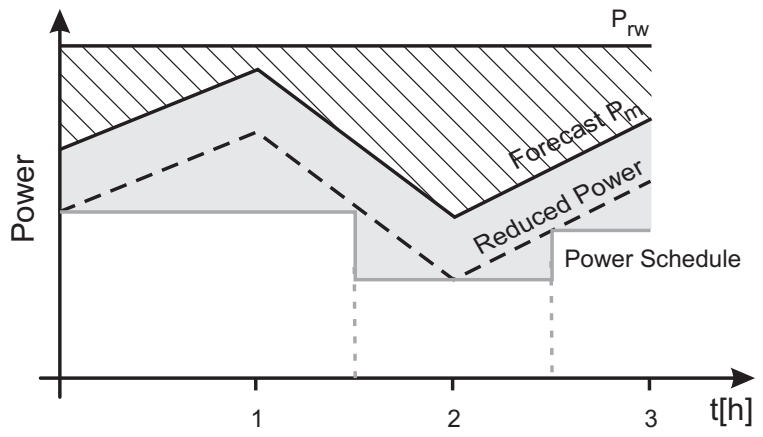

Fig. 4 The margin for the MIO

In most cases some wind power plants are in the position to be switched off completely and others drive with reduced power. It actually make sense to drive some wind turbines not with the maximum forecasted power of the $P_{m}$. So one has the possibility to compensate slight fluctuations of the wind farm as well as in the transmission system. Concerning the shadowing effects and the operating hours they have to be balanced against each other in order to make a decision. It depend on the priority of the wind farm owner but also on the economic efficiency which is not considered here.

The output of the MIO is a detailed power schedule for every single windmill including the maintenance for the whole wind farm in one time period.

\section{Resulting schedule}

In Figure 5 the flow-chart of the schedule with MIO is shown. First the power schedule is developed based on the input data of the power forecast. The output is the schedule for the next day.

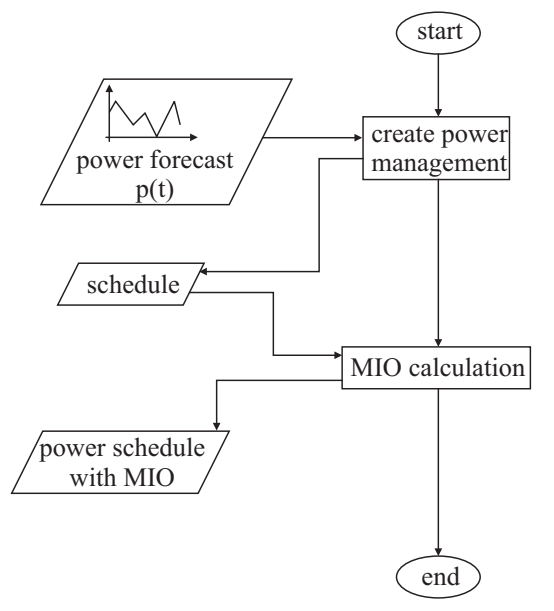

Fig. 5 Flow-chart power schedule with MIO

Following the MIO is added. The function of the MIO is to calculate how many windmills and which windmills have to be in service to fulfill the power schedule. The other windmills can bee out of service and therefore they save some hours concerning the maintenance. The aim is to have the maintenance of all windmills in the same time period. The output is a power schedule which contains the instruction for each windmill power output.

This procedure is done once a day for the next day and the power schedule is forwarded to the TSO.

\section{Controlled shutdown}

The controlled shutdown starts in case the wind speed exceeds $v_{w}>25 \mathrm{~m} / \mathrm{s}$. This procedure is a well organised shutdown.

The shutdown schedule is especially developed for offshore wind farms because the wind conditions are not applicable to onshore. It is assumed that offshore are no shadowing effects as well as no creation of swirls and an apparent wind front. In Figure 6 the design of the underlying wind farm is shown.

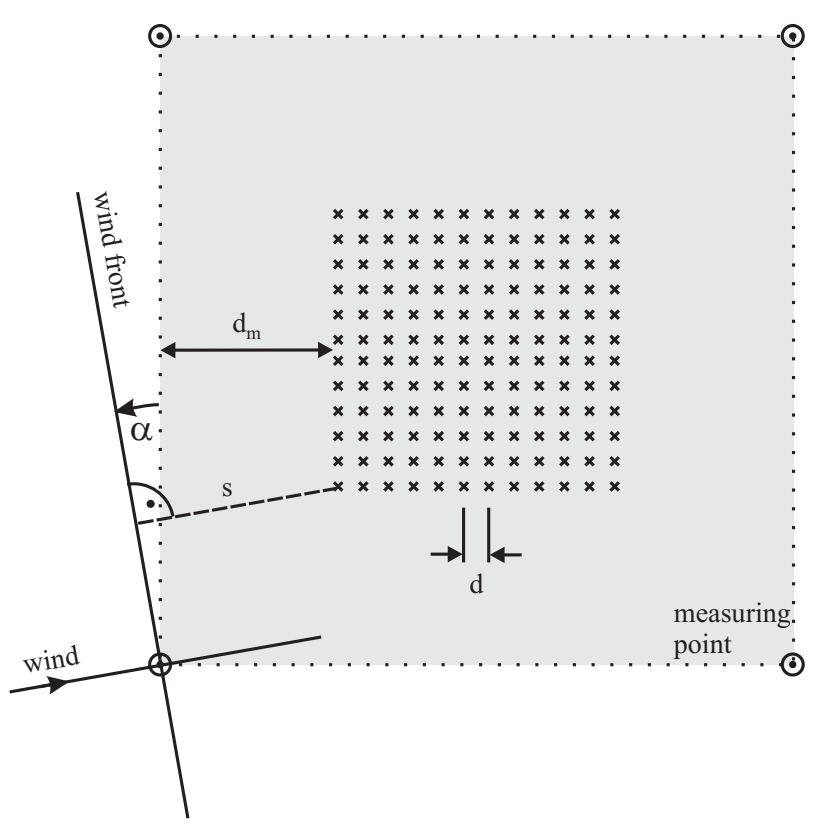

Fig. 6 Wind farm

\section{A. Development of the shutdown schedule}

The EMS controlled shutdown solution should contain the following requirements:

- The gradient of the power reduction during the shutdown should be small.

- The TSO should be informed about the shutdown as early as possible.

The measured data of the anemometers around the wind farm are evaluated. Some measuring points can be shared with other wind farms. The three windmills of the neighboring wind farm in Figure 1 represent such a common point for this example. As it can be seen in Figure 7 the measured data as well as the data of the German Meteorogical Service 
(DWD, Deutscher Wetterdienst) are imported and compared to avoid an unnecessary shutdown. It is proved if the status of the wind farm is active, that means the wind farm is running and if the wind speed is above $25 \mathrm{~m} / \mathrm{s}$. When both conditions are met a suitable schedule for the controlled shutdown is developed.

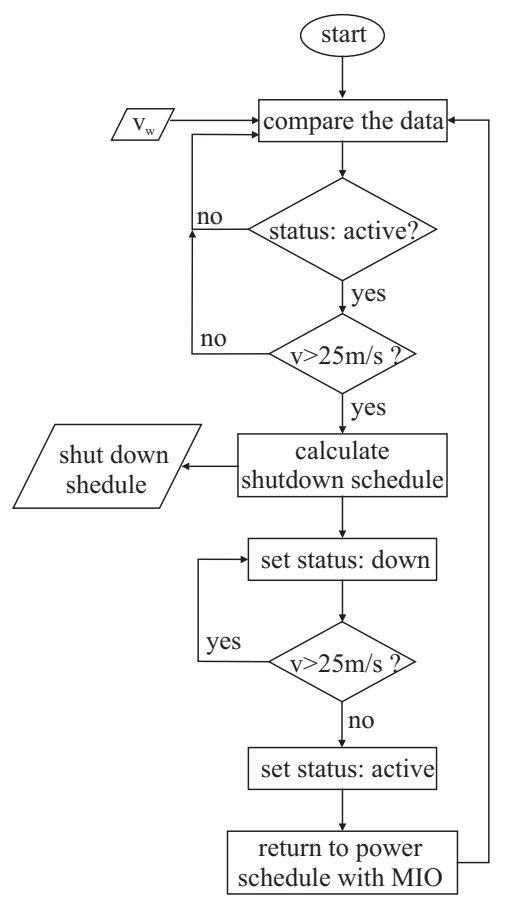

Fig. 7 Flow-chart shutdown schedule

The schedule contains information about the reduction intervals of the active power. The information about this procedure is given directly to the TSO. He has now all necessary information he needs to react to the coming controlled shutdown of the wind farm. According to the schedule, the set value for the power $P$ is derived and transmitted to the controllers of the UCS. The EMS of the whole wind farm supersedes the UCS. As a result, the wind farm shuts down as scheduled. The status of the wind farm after the shutdown will be set to down. The program returns to the power schedule. So as soon as the wind speed falls under $25 \mathrm{~m} / \mathrm{s}$ the wind farm cut-in again an the status of the wind farm turns to active.

This controlled shutdown has the priority. The power schedule is interrupted in case of a storm.

\section{B. Comparison to a shutdown without EMS}

The calculations of the power reduction are done for three different wind directions $\alpha=0^{\circ}, \alpha=20^{\circ}$ and $\alpha=45^{\circ}$. A distinct wind front is assumed here and no creations of swirls as well as no flurries which could shutdown single windmills out of order. Besides, the generated power of each windmill is equal before the shutdown and the power of the whole wind farm is $P_{w}$. This is realistic because usually before a storm there is enough wind to run all windmills of the wind farm.

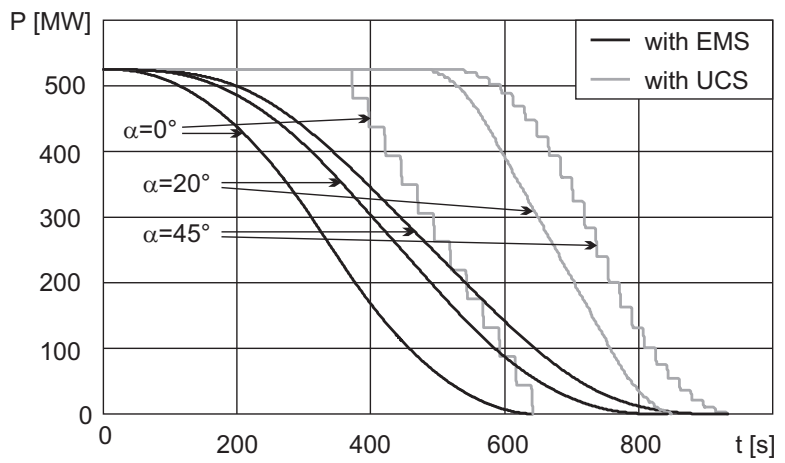

Fig. 8 Comparison of EMS and UCS at different angles of incidence

The time for the power reduction is much longer, compared to the UCS shutdown [2]. The application of the EMS results in a lower average gradient as well as in a lower maximum gradient of the reduction curve. In case of a wind speed of $v=30 \mathrm{~m} / \mathrm{s}$ and an angle of incidence of $\alpha=0^{\circ}$ the reduction of the power becomes

$$
\Delta P=22 \% \cdot \frac{P_{w}}{60 \mathrm{~s}}
$$

regarding the shutdown of a wind farm with a UCS. This value is reduced to an average

$$
\Delta P=10 \% \cdot \frac{P_{w}}{60 \mathrm{~s}}
$$

when using the EMS. For angles other than $\alpha=0^{\circ}$ this effect is even greater.

The comparison of the energy output by using the EMS is shown in Figure 9. The difference between the two procedures amount to a energy difference of about $34 \%$, if only the time inervall of the shutdown is considered. Annualized it doesn't carry wight which method is used.

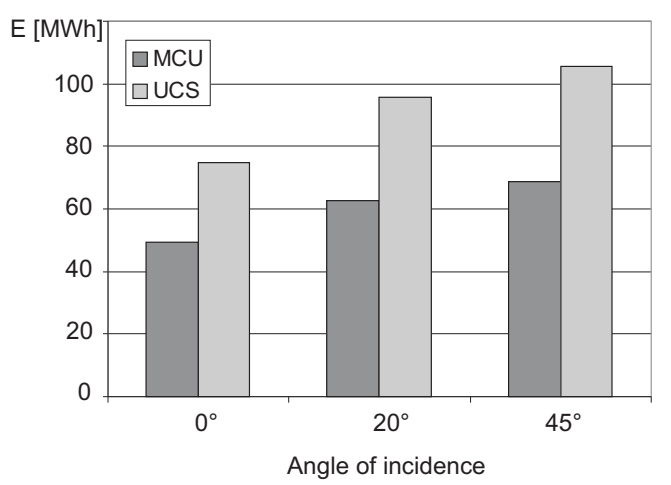

Fig. 9 Comparison of the resulting energy at different angles of incidence 


\section{System Management}

The system management contains the two just presented modules. In Figure 10 it is visualised that there is on the one hand the power schedule including the Maintenance Interval Optimization (MIO) and on the other hand the shutdown in case of to heavy wind.

The power schedule with MIO is a calculation that is done for the next 24 hours. That means the calculation takes place and in the next 24 hours the wind farm will be driven exactly concerning the power schedule with MIO. Eventually after 12 hours another calculation with the newest data for the forecast will be done just to update the schedule.

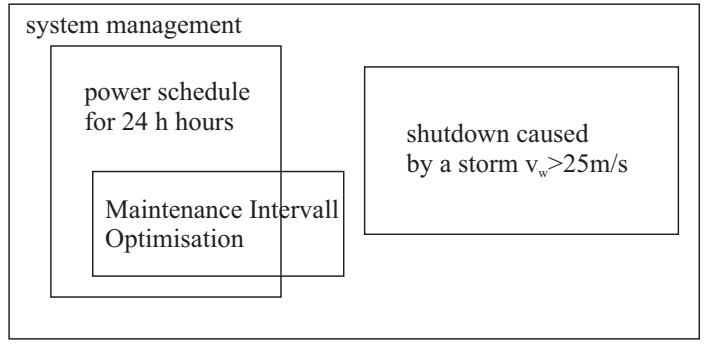

Fig. 10 Layout of the system management

During the time the wind farm is running after the schedule the shutdown part is continuously active. This part monitors the wind data all the time and in case of to heavy wind $(v<25 \mathrm{~m} / \mathrm{s})$ a shutdown schedule is calculated. In that case the running of the wind farm after the power schedule is interrupted and replaced by the shutdown schedule. When the wind farm is down it starts again running after the former power schedule as soon as the wind speed is reduced under $v<25 \mathrm{~m} / \mathrm{s}$ for at least 10 minutes.

\section{Conclusion}

Due to the fact that wind energy is one of the fastest growing markets and offshore wind farms will have capacities of usual base load power station, it is necessary to develop some methods to assist the TSO to control the power system. With the proposed energy management system (EMS) an additional tool can be applied to support the TSO.

The power schedule is a development especially to reduce the impact of fluctuating power on the system. The TSO has now almost all necessary information to react in an appropriate way, because there is less uncertainty in the load flow caused by the wind energy. It is now possible to make a unit commitment including the wind energy, thus reducing the need of primary control power.

The shutdown schedule is developed to shutdown the wind farm gradually and to inform the TSO before the beginning of the shutdown. Hence, the lack of generated power will be responded by the TSO in an appropriate way.

\section{References}

[1] U. Radke, H. Kühn, Der Einfluß von Windenergie auf das Verbundnetz, e\&i Heft 10.2003

[2] E. Hau, Windkraftanlagen, 2. Auflage Springer Verlag 1996

[3] Ch. Eping, J. Stenzel, Control of Offshore Wind Farms for a Reliable Power System Management, IREP Symposium, Bulk Power System Dynamics and Control - VI, Cortina d'Ampezzo, Italy, August 2004

[4] G.Maibaum, Wahrscheinlickkeitstheorie und mathematische Statistik. 2. Auflage, VEB Deutscher Verlag der Wissenschaften, Berlin 1980

[5] D. Goldberg, Genetic Algorithm in Search, Optimization and Machine Learning, New York: Addison Wesley, 1989

[6] B. Ernst, Prognose der Windleistung für größere Energieversorgungssysteme, VDITagung, Bochum, März 2001 